\title{
Germanica
}

\section{„Sie schmatzen und rülpsen, schlabbern und schlürfen" - Tischszenen in den aktuellen deutschen Bilderbüchern}

"They gorge and burb, slobber and slurp" - Table scenes in German picture books

"Ils mangent bruyamment, ils boivent bruyamment, ils éructent " - scènes de repas dans la littérature pour enfants contemporaine de langue allemande

\section{Oxane Leingang}

\section{(2) OpenEdition}

\section{Journals}

Édition électronique

URL : http://journals.openedition.org/germanica/3024

DOI : 10.4000/germanica.3024

ISSN : 2107-0784

\section{Éditeur}

Université de Lille

Édition imprimée

Date de publication : 30 décembre 2015

Pagination : 69-82

ISBN : 9782913857360

ISSN : 0984-2632

\section{Référence électronique}

Oxane Leingang, « „Sie schmatzen und rülpsen, schlabbern und schlürfen“ - Tischszenen in den aktuellen deutschen Bilderbüchern », Germanica [Online], 57 | 2015, Online erschienen am: 30 Dezember 2017, abgerufen am 06 Oktober 2020. URL : http://journals.openedition.org/germanica/ 3024 ; DOI : https://doi.org/10.4000/germanica.3024 


\title{
„Sie schmatzen und rülpsen, schlabbern und schlürfen“"- Tischszenen in den aktuellen deutschen Bilderbüchern
}

\author{
Oxane LEINGANG \\ Universität Frankfurt am Main
}

\section{Tischkonflikte in Heinrich Hoffmanns Der Struwwelpeter}

Eine sabotierte Mahlzeit und ein Suppenboykott: Zwei Paradebeispiele für eskalierende Konflikte am Tisch beschreibt Der Struwwelpeter oder lustige Geschichten und drollige Bilder (1845). Dieser Bilderbuchklassiker des Frankfurter Arztes und Psychiaters Heinrich Hoffmann bietet, ,eine Lesart an, die die bürgerliche (Essens-) Moral, wie sie auf eindringliche und über sich selbst hinausreichende Art in den moralischen Beispielgeschichten der Aufklärung und in abgeschwächter Form in jenen des Biedermeier inszeniert wird, untergräbt und einen Gegenangriff vom Kind aus startet"1. Die Rebellion der Kinder gegen Verhaltensdiktatur der bürgerlichen Tafelrunde, die

1. - Sonja Jäkel, Inszenierung des Essens in der Kinder- und Jugendliteratur. Aufklärung - Romantik-Biedermeier, Frankfurt am Main, Lang, 2015, S. 253. 
Hoffmann als Essensverweigerung und motorische Unruhe codiert, stört erheblich die repressive Atmosphäre des Familienidylls ${ }^{2}$.

Die Geschichte vom Zappel-Philipp erinnert durch die Ermahnungen zum Wohlverhalten an die Tischzuchten, die seit dem Mittelalter das Essgebaren regelten ${ }^{3}$. „Ob der Philipp heut still/Wohl bei Tische sitzen will?"4 fragt der Vater im gravitätischen Ton eines Erziehers, der für das Einhalten der Tischmanieren verantwortlich ist. Philipp, der ,gaukelt/ und schaukelt/und trappelt/und zappelt" ${ }^{\text {"5 }}$, fällt schließlich rücklings vom Stuhl und räumt nebenbei die Tafel ab, wie der Erzähler ironisch kommentiert. „In großer Noth“ 6 ist der Vater, der versucht, in allerletzter Sekunde die Katastrophe abzuwenden. Die Tischdecke unter seinen Fingern wirft Falten, die wie Kratzer aussehen. Wutentbrannt reißt er seine Arme in die Höhe und stampft mit einem Fuß auf den Boden (seine Körpersprache erinnert dadurch an das Gebaren des SuppenKaspars). Die Mutter verharrt in der Rolle einer schweigsam-hilflosen Zeugin. „Stumm blicket sie“7 auf das Machtspiel zwischen dem Vater und dem Sohn, das beim Tisch ausgetragen wird, und bestätigt damit die weibliche Inferiorität innerhalb der patriarchalen Gesellschaft ${ }^{8}$. Lediglich ihre Gestik ändert sich von der Resignation zur Bestürzung: Während sie in der ersten Szene ihre Brille wie ein Monokel hält, sichtlich um die Wahrung der Contenance bemüht, inspiziert sie im zweiten und dritten Bild mit Entsetzen das Ausmaß der Tischunordnung.

Ums Essen gebracht, sind die Eltern im Schlusstableau ,zornig“ eine Karikatur ihrer eigenen Erziehungsmaxime der Selbstkontrolle. „Das Ritual der Mittagsmahlzeit im Familienkreis“, so Könneker, „steht pars pro toto für die bürgerliche Ordnung schlechthin" "9, die hier durch das trotzige und letztendlich siegreiche Kind ins Lächerliche gezogen wird. Dressler polemisiert diesen Gestus der Verweigerung sogar als eine anarchische Jugendrevolte: „Der Zappel-Philipp steht in schneidendem Gegensatz zu seinen Eltern. Repräsentieren diese den konservativen, im

2. - S. auch Beate Zekorn-von Bebenburg, ,Wie sich Familien bei Tisch präsentieren. Eine Ausstellung zum Thema ,Familienessen' im Struwwelpeter-Museum“, in: Kinder- und Jugendliteraturforschung Frankfurt, 2014, Heft 1-2, S. 38-41.

3. - Vgl. Thomas Schürmann, Tisch- und Grußsitten im Zivilisationsprozess, Münster, Waxmann, 1994.

4. - Heinrich Hoffmann, Der Struwwelpeter oder lustige Geschichten und drollige Bilder, 100. Aufl., Frankfurt am Main, Literarische Anstalt von Rütten \& Loening, 1876, S. 18.

5. - Ebd.

6. - Ebd., S. 19.

7. - Ebd.

8. - Marie-Luise Könneker, Dr. Heinrich Hoffmanns „Struwwelpeter“. Untersuchungen zur Entstehungs- und Funktionsgeschichte eines bürgerlichen Bilderbuchs, Stuttgart, Metzler, 1977, S. 126.

9. - Ebd., S. 129. 
engen Kreis des Angestammten maßvoll genießenden Bürgersinn, so ist Philipp der an allem Bestehenden rüttelnde, ruhelose und zerstörende Skeptiker und Freigeist, ja Anarchist. Nicht nur vergällt dieser sich selbst den behaglichen Lebensgenuß, er entzieht auch seiner Umgebung, weiterhin das Gift seiner umstürzlerischen Lebensanschauung verspritzend, die Grundlage sicherer Existenz"10.

In der Geschichte vom Suppen-Kaspar boykottiert der pausbäckige Protagonist mit Vehemenz seine Suppe. „Ich esse keine Suppe! Nein! Ich esse meine Suppe nicht! Nein, meine Suppe esse ich nicht" 11 - eine rhythmisierte Essens- und Gehorsamsverweigerung, die zum Mitskandieren und -stampfen animiert ${ }^{12}$. Mehr noch: Sie ist lesbar als die erste kinderliterarische Beschreibung der Anorexie ${ }^{13}$. Im Gegensatz zur Geschichte vom Zappel-Philipp wird hier eine einsame Tischszene in Abwesenheit der Erzieher dargestellt. Durch die hermetische Abschottung wird Kaspar von der Gesellschaft isoliert; seine Proteste verhallen ungehört. Erst diese pädagogisch-literarische Versuchsanordnung macht den Jungen zum Exempel. Auf kindliche Renitenz reagieren seine Eltern mit kinderfeindlicher Härte: „Das unpersönliche Arrangement verströmt eine Indifferenz nach dem Motto ,Friss oder stirb', das Kaspars Aufbegehren ohnmächtig macht“, so Jäkel ${ }^{14}$. Sein radikaler, im Zeitraffer präsentierter Gewichtsverlust ist eine ,katastrophenkomische Inszenierung" 15 , die durch ihre Überspitzung den pädagogischen Impetus konterkariert. Vom rundlichen Knaben bleibt im vierten Bild ein wütend-stampfendes „Fädchen“16 übrig. Das fünfte Bild zeigt sein Kindergrab mit einem Kreuz und einer Terrine als Grabschmuck - eine Aufforderung zum folgsamen Essen, die kaum drastischer ausfallen konnte. Auch wenn es sich auf den ersten Blick um eine moralische Warngeschichte handelt, befriedigt das Ausmalen des Trauerprozesses, wie Insa Friese scharfsinnig für den Subtext attestiert, „die kindlichen Rachegelüste an den unnachgiebigen Eltern. Die tiefe Trauer und Bestürztheit der Eltern zu imaginieren, die diese beim erbärmlichen Tod des standhaften Speisenverweigerers erleiden, hat ja durchaus etwas

10. - Dressler zit. nach Könneker, ebd., S. 129 f.

11. - Hoffmann, Struwwelpeter, a.a.O., S. 17.

12. - Jäkel, Inszenierungen, a.a.O., S. 246.

13. - Tanja Rudtke, Kulinarische Lektüren. Vom Essen und Trinken in der Literatur, Bielefeld, transcript, 2014, S. 167.

14. - Ebd., S. 244.

15. - Rüdiger Steinlein, „Kinderliteratur und Lachkultur. Literarhistorische und theoretische Anmerkungen zu Komik und Lachen im Kinderbuch“, in: Hans-Heino Ewers (Hg.): Komik im Kinderbuch. Erscheinungsformen in der Kinder- und Jugendliteratur, Weinheim, Juventa, 1992, S. 11-32, hier S. 19.

16. - Hoffmann, Struwwelpeter, a.a.O., S. 17. 
Entlastendes und Entschädigendes für ein Kind, das erbarmungslos zum Essen gezwungen wird“17.

\section{Die modernen Speisenverweigerer}

„Ich mag keine Suppe!“ konstatiert der kindliche Ich-Erzähler gleich am Anfang des gleichnamigen Bilderbuches aus dem Jahre 2009. Der Text stammt aus der Feder der Berliner Architektin Gesa Glück. Die Illustrationen steuerte ihr Vater, der renommierte Kasseler Cartoonist Gerhard Glück, bei. Angesichts postmoderner Grenzenüberschreitung zwischen Hoch- und Trivialkultur und Auflösung fester Adressatenentwürfe wundert es kaum, dass ein preisgekrönter Künstler wie Glück eigene und fremde (Bilder-)Bücher illustriert. Im Zuge des von W.J.T. Mitchell ausgerufenen ,pictorial turns“ - des Wechsels von der Text- hin zur Bildzentrierung der westlichen Gesellschaft durch das Aufkommen des Fernsehens, der Reklame, der Videospiele und des Internets - wurden Bilderbücher als visuelle (Kunst-)Objekte aufgewertet und avancierten aufgrund ihrer Mehrdeutigkeit zur Lektüre von Erwachsenen ${ }^{18}$.

Typographisch ahmt der Titel in krakeligen Großbuchstaben eine Kinderschrift nach und erhält eine zusätzliche Emphase durch das Ausrufezeichen. Wie bei Hoffmanns Unglücksgeschichten ist das Text-Bild-Verhältnis komplettierend, d. h. die piktorale und verbale Zeichenebenen bestätigen und ergänzen sich gegenseitig, wodurch die für die Bilderbücher charakteristischen synästhetischen Effekte entstehen ${ }^{19}$. Die kräftigen Grün- und Blautöne dominieren die reduzierte, dunkle Farbpalette; für Kontraste sorgt das sparsam verwendete Rot. In verschiedenen Schattierungen taucht das matt-grünliche Hellgelb - die Farbe der Suppe - nicht nur im Titel und im Vorsatz des Buches, sondern leitmotivisch in jeder Illustration auf. Genau wie sein berühmter Vorgänger heißt dieser Suppenboykotteur Kaspar. Der Duft der Kartoffel-Lauch-Suppe steigt dem Jungen im Hausflur in die Nase und verdirbt ihm sofort die Laune ${ }^{20}$. Freitags gibt es immer eine aufgewärmte Suppe als Behelfsessen: Für Kaspar ist es eine einsame Mahlzeit, denn die berufstätige Mutter ist außer Haus und der am

17. - Insa Friese, „Ein Klassiker am Ausgang seiner Epoche. Heinrich Hoffmanns ,Der Struwwelpeter““, in: Bettina Hurrelmann (Hg.), Klassiker der Kinder- und Jugendliteratur, Frankfurt am Main, Fischer, 1995, S. 358-379, hier S. 369.

18. - Zur Öffnung von Adressatenkonzepten s. auch Gabriele v. Glasenapp, Kinder- und Jugendliteratur, Paderborn, Schöningh, 2010, S. 174-182.

19. - Lawrence R. Sipe, „How Picture Books Work: A semiotically framed theory of text-picture relationships", in: Children's Literature in Education, 1998, 29, S. 97-108.

20. - Gesa Glück/Gerhard Glück, Ich mag keine Suppe! Oldenburg, Lappan, 2009, Seiten nicht paginiert. 
Tisch sitzende Vater geistig abwesend und abweisend. Schweigend isst der Vater seine Suppe, ganz in die Zeitung versunken, die normalerweise am Tisch streng verboten ist - eine heimliche Rebellion gegen die weibliche Autorität. Das Autorenduo demaskiert hier die „typische Verfallstruktur einer familialen Entfremdungssituation“21: Die Faceto-Face-Kommunikation am Tisch, die aus Protesten und reflexartigen Beschwichtigungen besteht, ist auf ein Minimum reduziert. Auch die kindliche Verzögerungstaktik des Trödelns und Tagträumens sowie das listige Versprechen eines Desserts seitens des Vaters gehören zu jenen zentralen Tischritualen, die hier durchgespielt werden. Die Kraftprobe zwischen Vater und Sohn, die bei Hoffmann am Tisch ausgetragen wird, bleibt aus.

Zunächst ungeduldig, dann genervt reagiert der Vater, als der Sohn in seinem Teller ein winziges U-Boot erspäht, das er durch das rasche Auslöffeln der Suppe trocken legt. Die havarierte Besatzung Geologen, Ozeanographen und Matrosen des pazifischen Inselstaats Minimania - bedarf Kaspars Hilfe, um auf dem Seeweg in die Heimat zurückzukehren. Nebenbei werden die Reste der verhassten Suppe, die als ein „gelblicher, sämiger Brei“ im Textverlauf mehrmals als Kleinkindernahrung abqualifiziert wurde, von der ausgehungerten Crew zu einer Köstlichkeit aufgewertet. Als das U-Boot beim Herunterfallen in tausend Stücke zersplittert, kommt das kitschige Erbstück, ein Modell-Zweimaster als Flaschenschiff, zum Einsatz auf der Spree. Der Essenszwang endet hier nicht wie bei Hoffmann tödlich, sondern wird im Rahmen einer Abenteuergeschichte jenseits der elterlichen Kontrolle in eine Rettungsaktion umcodiert. Die Suppe wird dadurch zum Therapeutikum gegen Langeweile am Tisch und zur Inspirationsmetapher.

Die Miniaturisierung der Mannschaft, diese für das Phantastische typische Thematisierung des Kleinen, ${ }^{22}$ ist ein Identifikationsangebot für junge Leser. Kaspar ist zwar einerseits den Erwachsenen körperlich unterlegen und muss ihre Vorschriften befolgen. Anderseits ist er aufgrund seiner Größe und seines Ideenreichtums für die Besatzung des U-Boots von solch großer Bedeutung, sodass er sogar mit einem für alle unsichtbaren Verdienstorden bedacht wird. Er wird zum Geheimnisträger des Phantastischen, das er durch Verstecken und Verschleiern vor den Zugriffen seiner neugierigen Eltern schützt. „,Aber, Kaspar, du weißt doch, morgen ist Freitag', sagte meine Mama. Und mein Papa ergänzte:

21. - Alois Wierlacher, Vom Essen in der deutschen Literatur. Mahlzeiten in Erzähltexten von Goethe bis Grass, Stuttgart, W. Kohlhammer, 1987, S. 158.

22. - Vgl. Mary Nortons The Borrowers (1952) oder Astrid Lindgrens NilsKarlsson Pyssling (1949) (dt. Nils-Karlsson Däumling, 1952). 
,Und Freitag gibt es wie immer eine Suppe'“23. Die letzten Sätze sind somit nicht nur als eine kulinarische Drohung, sondern auch als eine Verheißung neuer Abenteuer zu verstehen.

Mit Lola rast legt Wilfried von Bredow 2005 eine weitere Struwwelpetriade ${ }^{24}$ vor. Mit diesem Bilderbuch debütierte der emeritierte Professor für Außen- und Sicherheitspolitik von Bredow als Kinderbuchautor. Bereits der Untertitel und andere schreckliche Geschichten mit drolligen Bildern rekurriert auf den berühmten Prätext. Auch in diesem Bilderbuch ist die Doppeladressierung 25 bereits im Paratext ${ }^{26}$ markiert: „,Sieben schaurig-schöne Geschichten für mutige Kinder und ängstliche Erwachsene - oder umgekehrt", heißt es auf dem Cover. Strukturell an Hoffmann angelehnt, werden in sieben Episoden zahlreiche Erziehungsprobleme - Medienkonsum, Schönheitswahn, Unordnung, Ungehorsam - durchgespielt und in dramatischen Showdowns, der Logik der Warngeschichte folgend, „katastrophenkomisch" zur Eskalation gebracht. Wie bei Hoffmann überwiegt auch hier die belustigende Unwahrscheinlichkeit des Unglücks. Am Ende bleiben die tief bestürzten Eltern, deren Ermahnungen von den Kindern abprallten und deren Rettungsaktionen scheitern; lesbar entweder als ein empathisches Plädoyer für folgsames Verhalten oder als eine Befriedigung kindlicher Racheimpulse.

So rast die umtriebige, titelgebende Lola auf ihrem Laufrad durch große Pfützen an den manierlichen Passanten vorbei, gegen die Mülltonne gleich in den gut sortierten Obststand. Die motorische Unruhe von Zappel-Philipp wird hier mechanisiert und ins Extreme gesteigert; die Straßen als Gefahrenzonen inszeniert. Auch die Tischordnung in einem Straßencafé bringt Lola ,jauchzend“ durcheinander, als sie dem Kellner ungebremst ans Bein „rattert“, der just in dem Moment Eis und Limonade serviert: „Vorn dem Kellner tropft das Eis. Die Weste ist nun nicht mehr weiß“, heißt es lakonisch ${ }^{27}$. Lolas Erkundungs- bzw. Amokfahrt findet ein jähes Ende. Sie wird von einem Lastwagen ,plattgedrückt" und zusammen mit ihrem geliebten Gefährt beerdigt ${ }^{28}$. Ihr Unfalltod bestätigt jene düstere Prophezeiung, die ihre besorgte Mutter bereits am Anfang aussprach: „An der Kreuzung und

23. - Letzte Seite, nicht paginiert. 2012.

24. - Karl Riha, Struwwelpeter und Struwwelpetriaden, Frankfurt am Main, Lang,

25. - Zum Phänomen der Doppeladressierung in der Kinder- und Jugendliteratur vgl. Hans-Heino Ewers, Literatur für Kinder und Jugendliche. Eine Einführung, 2. Aufl., Paderborn, Fink, 2012, S. 57-67.

26. - Zum Paratext in der Kinderliteratur ebd., S. 44-56.

27. - Wilfried von Bredow, Lola rast und andere schreckliche Geschichten mit drolligen Bildern von Anke Kuhl, 2. Aufl., Leipzig, Klett Kinderbuch, 2009, S. 7.

28. - Ebd., S. 8. 
bei rot/halte an, sonst bist Du tot“"29. Mit der Todesdrohung, welche die Vermeidbarkeit des Risikos suggeriert, rekurriert von Bredow wie bereits Hoffmann auf die Unglücksgeschichten der aufgeklärten Kinderliteratur, die sich auf Verstümmelungen, Verletzungen und Tod von kindlichen Übeltätern kaprizierte ${ }^{30}$. Auch die Verkehrserziehung als Einübung in die Straßenverkehrsordnung wird hier im Rahmen einer drastischen Abschreckungspädagogik mithilfe der Deformationskomik ins Groteske gesteigert; darüber hinaus wird die Ablösungsgeschichte ironisiert, die dieser Anfängerliteratur inhärent ist.

Die Geschichte Die schöne Anna-Lena nimmt nicht nur das geschlechtsspezifische Schönheitsideal, das Modediktat und das (kindliche) Konsumverhalten aufs Korn, sondern führt auch das traditionsreiche Erziehungsziel ad absurdum, das auf die Hemmung der Gesamtmotorik abzielte. In Spitze und Rüschen gekleidet, verharrt Anna-Lena vor dem Spiegel und imitiert die Bewegungen ihres Vorbilds - einer Puppe -, bis sie schließlich selbst zu einem anthropomorphen Artefakt wird. Diese erdachte Geschichte bestätigt damit die These der Entwicklungspsychologin Insa Fooken: „In gewisser Weise ,produziert' die Puppe das mit ihr spielende Kind und beeinflusst dessen Selbst- und Körperwahrnehmung, spiegelt ihm Ähnlichkeiten und/oder Anderssein wider, sagt ihm in ihrer äußeren Puppen-Körperlichkeit, wer dazugehört und wer abweicht" ${ }^{* 31}$. Die Puppe fungiert hier nicht nur als Projektionsfläche für kindliche Sehnsüchte, sondern als eine Kippfigur zwischen Lebendig- und Totsein: „Der Schein des Lebens macht sie [die Puppe] zu einer attraktiven Spielgefährtin, der Schein des Todes [...] gibt ihr den Charakter einer heimlichen/unheimlichen Grenzgängerin und einer Allegorie des Todes“32. Der Erzähler beschreibt die tödliche ,Verpuppung' Anna-Lenas, die am Ende der Materialität des Spielzeugs und dessen Attraktivitätsstandards entspricht: Hölzern knacken ihre Gliedmaßen, bis sie zu einer starren Kinderleiche wird; das sorgfältig eingeübte Lächeln gerinnt auf ihren Lippen zu einer Grimasse:

Beim Essen sitzt sie steif am Tisch.

Im Rücken knackst es fürchterlich.

Sie hebt die Hand mit letzter Kraft

und greift nach ihrem Apfelsaft.

29. - Ebd., S. 5.

30. - Dieter Richter, Das fremde Kind. Zur Entstehung der Kindheitsbilder des bürgerlichen Zeitalters, Frankfurt am Main, S. Fischer, 1987, S. 81-83.

31. - Insa Fooken, „Mehr als ein Ding: Vom seelischen Mehrwert der Puppen“, in: Insa Fooken/Jana Mikota (Hgg.), Puppen, Menschenbegleiter in Kinderwelten und imaginären Räumen, Göttingen, V\& P, 2014, S. 44.

32. - Gundel Mattenkott, „Heimlich-unheimliche Puppe: Ein Kapitel zur Beseelung der Dinge“, in: ebd., S. 36. 


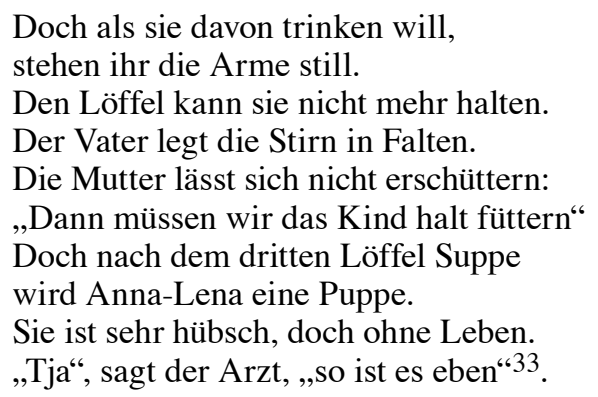

Der alterstypische Bewegungsdrang, bei Hoffmann in motorische Unruhe übersteigert, verlangsamt sich hier zum Stillstand. ZappelPhilipp ist Anna-Lenas Kontrastfigur. Der repressive Esszwang wird zur fürsorglichen Zwangsernährung. Zur dekorativen Marionette denaturiert ist Anna-Lena am Ziel ihrer ästhetischen Selbstoptimierung: Als Ebenbild ihrer Puppe ist sie endlich „sehr hübsch, doch ohne Leben“ und kann nicht mehr zum Spielen mit den anderen Kindern im Hof gezwungen werden ${ }^{34}$.

\section{Tischszenen als Integrationsrituale}

Dass das gemeinsame Essen nicht nur die Sozialität demonstriert, sondern auch eine (Kinder-)Gemeinschaft rituell stabilisiert, wird in Der böse Heinrich vorgeführt. In Anlehnung an Hoffmanns Die Geschichte vom bösen Friederich problematisiert von Bredow kindliche Aggressivität und Devianz. Im Struwwelpeter malträtiert Friederich anfangs nur Insekten und Tiere: Er reißt den Fliegen die Flügel raus und erschlägt den Singvogel im Käfig. In der nächsten Eskalationsstufe zerstört er das Mobiliar des bürgerlichen Haushalts und peitscht sein Kindermädchen aus. Friederich wird schließlich von einem Hund gebissen und muss das Bett hüten.

Auch bei von Bredow werden die Missetaten des kindlichen Aggressors drastisch ausgemalt. Im Gegensatz zu Hoffmann verlagert sich die Handlung ins Grüne. Der Miniatursadist Heinrich tyrannisiert Kinder auf dem Spielplatz: Er zieht sie an den Haaren, stößt sie von der Wippe herunter und ist sogar für Knochenfrakturen verantwortlich. So wundert es nicht, dass an seinem Geburtstag die Gratulanten fernbleiben. In der Kinderkultur hat der Geburtstag als „Legitimation für kleine Macht- und Kraftproben“ 35 einen besonders hohen Stellenwert, weil das

33. - Bredow, Lola, a.a.O., S. 15

34. - Ebd.

35. - Regine Falkenberg, Kindergeburtstag - Eine Brauchstudie über Kinder und ihr Fest, Inaugural-Dissertation zur Erlangung der Doktorwürde des Fachbereichs 
Kind ins Zentrum des familiären Geschehens gerückt und ihm dadurch für genau einen Tag Bestimmungsrecht und Handlungsautonomie suggeriert wird. Zwei Festelemente erweisen sich dabei als essentiell: Geschenke und Kindergesellschaft. Bei von Bredow kommt es allerdings zur Umkehrung der Verhältnisse. Heinrichs Opfer strafen ihn mit Desinteresse und missachten den „Kinderbeschertermin“36:
Als er Geburtstag feiern will, bleibt es bei ihm zu Hause still.
Denn keiner kommt. Er ärgert sich.
Er wird zum wilden Wüterich!
Und schmeißt ganz außer Rand und Band die schöne Torte an die Wand ${ }^{37}$.

Die einsame Tischszene an seinem Ehrentag avanciert zum Katalysator für einen moralischen Regenerationsprozess. Wutentbrannt will Heinrich seine Zurückweisung körperlich ausagieren. Nachdem zunächst die Torte als symbolisches Festrequisit zum Ventil für seinen Zorn wird, eilt er zum Spielplatz. Doch als er keinen zum Verprügeln findet, wird ihm seine Einsamkeit bewusst. Seine Gewaltbereitschaft schlägt in Hilflosigkeit um. Nach einem kathartischen Heulkrampf schreit er seine Selbstkritik und seinen Besserungswunsch in die Welt hinaus: „Ich hau nicht mehr! Es tut mit leid! Lasst mich bitte nicht allein! Will nicht der böse Heinrich sein!" 38 Während bei Hoffmann der bissige Hund als eine ,natürliche' Strafinstanz auftritt, zeigt von Bredow in einer introspektiven Bilanzierungsphase die Läuterung eines Bösewichts. Die Ursachen des aggressiven Verhaltens bleiben auch hier im Dunkeln.

Das Tischrundenkonzept - die befriedende und gemeinschaftsstiftende Wirkung von Mahlzeiten - zeigt die letzte Doppelseite des Bilderbuchs Wann gehen die wieder? (2010) der Regisseurin, Drehbuchautorin und Illustratorin Ute Krause ${ }^{39}$. Mithilfe des Märchenpersonals präsentiert Krause die typischen Probleme der Patchworkfamilien und bietet als Lösungsvorschlag ein Harmoniebild des Sichzusammenfindens beim Essen, denn ,keine andere Form der Vergemeinschaftung bindet so stark wie die des gemeinsamen

Gesellschaftswissenschaften und Philosophie der Philips-Universität Marburg/Lahn, 1982, S. 1.

36. - Ebd., S. 134.

37. - Bredow, Lola, a.a.O., S. 16.

38. - Ebd., S. 18.

39. - Ute Krause, Wann gehen die wieder? Berlin, Bloomsbury Kinderbücher \& Jugendbücher, 2010, Seiten nicht paginiert. 
Tisches - man denke nur an die sagenhafte Tafelrunde des König Artus" ${ }^{40}$. Bei Krause ist es eine karierte Picknickdecke, die idealtypisch die Gleichheit der Essenden verdeutlicht und ihre Unterschiede nivelliert. Die Scheidung der Räuber-Eltern führt zur Duplizierung von Wohnräumen und Festen; das nomadische Hin und Her nährt die nostalgische Sehnsucht nach familiärer Einheit. Als der Vater sich erneut verliebt, gerät die Welt der Räuber-Kinder endgültig aus den Fugen. Zum Missfallen der Kinder ziehen seine neue Flamme, eine Prinzessin, und ihre Kinder zu ihnen und stören das ohnehin defizitäre Familienidyll. Bereits die titelgebende Frage entindividualisiert die Eindringlinge und unterminiert das Gastlichkeitsritual. Die RäuberKinder hecken eine Intrige aus, um den Familienzuwachs loszuwerden. Auf den Verlust seiner Geliebten reagiert der Vater mit Depression. Die Kinder langweilen sich, weil sie keinen mehr zum Ärgern haben. Ein opulentes Versöhnungsessen in der Freilichtkulisse steht symbolisch für den erfolgreichen Integrationsprozess - der Räuber, der Prinzessin und des Drachens, der als der neue Freund der Räuber-Mutter die Familienkonstellation (vorläufig) komplettiert.

\section{Grobianische Monster der Die Olchis-Serie}

Die äußerst populäre kinderliterarische Buchreihe Die Olchis von Erhard Dietl arbeitet mit der Fiktion der Vorbildlichkeit von Unsitten und entwirft dadurch eine alternative kulturelle Ordnung. Zuerst 1990 als Erstlesebuch publiziert, ist die Serie inzwischen auf 30 Titel angewachsen, zu denen sechs Bilderbücher und 24 Kinderromane zählen. Jährlich erscheint ein Buch, das multimedial vermarktet wird. Bis 2010 belief die Gesamtauflage auf mehr als drei Millionen Exemplare. Nach einem eigenen Theaterstück und Musical gibt es seit 2011 alle zwei Monate am Kiosk das Olchi-Magazin. Erhard Dietl, der bislang über 150 Kinderbücher geschrieben hat, ist ein ,,autorstator“41: Er ist der Schöpfer, Autor und Illustrator. Als Singer-Songwriter vertonte er einige „olchige“ Nonsense-Gedichte, die später in den KinderfunkHitparaden ganz oben rangierten.

Kurze Episoden beschreiben das bizarr-chaotische Treiben einer lärmenden Drei-Generationen-Familie. Die Olchis sind grüne, gehörnte Phantasiewesen, die auf einer Mülldeponie wohnen, Ordnung hassen und sich in allerlei Abenteuer stürzen. Mehr noch: Die Olchis sind

40. - Eva Barlösius, Soziologie des Essens. Eine sozial- und kulturwissenschaftliche Einführung in die Ernährungsforschung, 2. völlig überarb. und erw. Aufl., Weinheim/ München, Juventa, 2011, S. 173.

41. - Martin Salisbury, „The Artist and the Postmodern Picturebook”, in: Lawrence R. Sipe/Sylvia Pantaleo (Hgg.), Postmodern Picturebooks. Play, Parody, and SelfReferentiality, New York/Abington, Routledge, 2008, S. 22-40, hier S. 23. 
eine Rotte von Rüpeln. Sie sprechen eine originell-ordinäre, expressive Sprache voller kühner Neubildungen. Neben jeder Menge Slapstick und den häufigen frechen Schimpftiraden steigern vor allem die körperbezogenen Aktionen den anarchischen Spaß ins Extreme: „Olchi-Oma [...] rülpst so kräftig, dass ein paar Fliegen tot zu Boden fallen“"42. Eine heftige Neigung zu Eruktation und Flatulenz fungiert hier als Teil des Körperdramas ${ }^{43}$, das sich in der Kinderliteratur nur auf Einverleiben und Verdauen beschränkt. Mit komisierender Direktheit zelebriert diese Serie tabuisierte Leiblichkeit und körperliche Komik, was für die kindlichen Leser rezeptionspsychologisch besonders reizvoll ist ${ }^{44}$. In ihrer Zwanglosigkeit erscheinen die Olchis nicht nur als eine emanzipatorische Verkörperung des Lustprinzips, sondern als eine Projektionsfläche für Regressionsphantasien jenseits des Peinlichkeitsgefühls. Ihre Regelverstöße machen die Regel erst sichtbar. Ähnlich wie Hoffmann präsentiert Dietl eine Rebellion gegen die Zivilisierung, die sich unter anderem in der Kultur des Karnevals sowie im SchlaraffenlandKomplex finden lässt - gegen das Sauberkeitsdiktat, das Arbeitsethos und die Tischetikette.

Mit der Spiegelung der Gesellschaft schreibt sich die Olchis-Serie subtextuell in die satirische Tradition der verkehrten Tischzuchten ein, unter denen Friedrich Dedekinds Grobianus De morum simplicitate (1549) und Hans Sachs' Die verkehrt dischzuecht Grobiani (1563) eine prominente Rolle einnehmen. Diese parodistische Umkehrung der höfischen Wohlerzogenheit ist weder eine Reaktion auf besonders tadelnswerte Tischsitten, noch eine kulturpathologische Chronik des Sittenverfalls, sondern vielmehr lesbar als ein intertextuelles Spiel mit den Konventionen des Genres ${ }^{45}$. Um als ein Grobian zu gelten, so die ironische Negativdidaxe, benehme man sich möglichst „,ungesittet und tölpelhaft: schmatze, stoße den Becher um, [...] stopfe sich den Mund voll, rede mit vollem Mund [...]. Man rülpse und furze, schneuze sich oft, stochere in den Zähnen herum, greife unter sein Gewand und suche

42. - Erhard Dietl, Die Olchis. Allerhand und mehr, Hamburg, Oetinger, 2003, S. 12.

43. - Zum Körperdrama vgl. Michail Bachtin, Literatur und Karneval. Zur Romantheorie und Lachkultur. Aus dem Russischen übersetzt und mit einem Nachwort versehen von Alexander Kaempfe, Frankfurt am Main, Fischer, 1990, S. 47-60.

44. - Zu Karnevalismen in der Kinderliteratur als Manifestationen des MateriellPhysischen s. Maria Lypp, „Lachen beim Lesen. Zum Komischen in der Kinderliteratur (1986)“, in: Dies.: Vom Kaspar zum König. Studien zur Kinderliteratur, Frankfurt am Main, Lang, 2000, S. 87-99, hier S. 93.

45. - Christiane Vogt, Forschungen zu den selbständigen deutschen Tischzuchten des Mittelalters und der frühen Neuzeit, Inaugural-Dissertation zur Erlangung des Akademischen Grades eines Dr. phil., vorgelegt dem Fachbereich 13 - Philologie I der Johannes Gutenberg-Universität Mainz, 1995, S. 285. 
nach Flöhen" 46 . So wundert es nicht, dass der grobianische Normbrecher aufgrund seiner exzeptionellen Negativität zum Prototyp vieler Missetäter der Kinderliteratur avancierte ${ }^{47}$. Somit reihen sich die Olchis in das kinderliterarische Horrorkabinett ein, das von ,grobschlächtigen, schrägen, schielenden und vor allem verfressenen Gesellen, [...] von Dickbäuche(n), Gierschlunde(n) und Großmäuler(n)" bevölkert ist ${ }^{48}$.

Die Olchis kennen keine Tabus und halten Matsch, Kalk und Metallschrauben für Delikatessen, was die strikte Unterscheidung zwischen Genießbarem und Ungenießbarem auf den Kopf stellt. Die physiologisch-objektiven Kategorien der Giftigkeit und Unverdaulichkeit werden in diesem gustatorischen mundus inversus positiv codiert. Die für den Menschen gesunde Nahrung verursacht bei den Olchis eine akute Lebensmittelvergiftung, die nur mit einer Matschsuppe kuriert werden kann. Mit der Erschaffung einer essbaren Landschaft wird zum einen auf die orale Phase der Kleinkinder rekurriert, in der sie alles für essbar und trinkbar halten, und somit auf die Chiffre für die kindliche Weltaneignung. Zum anderen wird auch der alte Schlaraffenland-Topos aufgegriffen, der in der Kinderkultur prominent vertreten ist ${ }^{49}$. „Die ,eßbare Welt'“, resümiert Dieter Richter, ,ist, so oder so, aus primärer Lust oder aus quälendem Hunger, der Traum dessen, der vor allem aus Mund und Bauch besteht" ${ }^{*}$. In ihrer Esslust machen die Olchis auch vor Gebäuden nicht halt. So knabbern sie bei einem Familienausflug nach Paris nicht nur die Eisenstangen des Eiffelturms an, sondern bringen die Stahlträger aus ihrer Aufhängung 51 , ehe sie sich nach Italien trollen, um den Schiefen Turm von Pisa noch rechtzeitig gerade zu rücken ${ }^{52}$. „Mit ihren Grotesken, Grobianismen und dem kruden Humor, der das Ernste

46. - Ebd., S. 279.

47. - Könneker, Struwwelpeter, a.a.O., S. 27-29. Dazu auch Mareile Oetken, „Monströs, maßlos und komisch. Der Grobian als fantastische Figur in Kinderliteratur und Bilderbuch“, in: Ute Dettmar/Mareile Oetken/Uwe Schwagmeier (Hgg.), SchWellengänge. Zur Poetik, Topik und Optik des Fantastischen in Kinder- und Jugendliteratur und -medien, Frankfurt am Main, Lang, 2012, S. 215-230.

48. - Ute Dettmar, Scherz, List, Rache. Formen und Funktionen des Komischen in der Kinderliteratur. Oldenburger Universitätsreden, Vorträge, Ansprachen, Aufsätze, Oldenburg, BIS-Verlag, 2009, S. 22.

49. - Ute Dettmar, „Glück im Überfluss? Zur kinderliterarischen Besetzung des Schlaraffenlandes“, in: Caroline Roeder (Hg.), Topographien der Kindheit: Literarische, mediale und interdisziplinäre Perspektiven auf Orts- und Raumkonstruktionen, Bielefeld, transcript, 2014, S. 347-362.

50. - Dieter Richter, Schlaraffenland. Geschichte einer populären Phantasie, Frankfurt am Main, Anton Hain, 1989, S. 30.

51. - Dietl, Die Olchis, a.a.O., S. 144-150.

52. - Ebd., S. 153. 
permanent ins Lächerliche auflöst" 53 , werden hier die schlaraffischen Darstellungen der populären Lachkultur zitiert.

Mit genussvollem Entsetzen beschreibt der Erzähler nicht nur die Mahlzeiten der Olchis, sondern auch die Rezeptur der Speisen. So setzt sich ihr Leibgericht - der ,Schumuddeltopf mit Soße“ aus Zahnpasta, zerstoßenem Glas, Tinte, Gips und toten Fliegen zusammen ${ }^{54}$. Die Menüfolge des Festessens parodiert und ironisiert die ästhetische Sensibilität sowie die distinguierte Stilisierung der gehobenen Küche: „Blauer Draht auf Papiersalat (aus altem Buch) garniert mit Schneckenhaus und nassem Sand; Scherben von grüner Flasche gehackt in Topflappen mit dunkler Soße aus dem Erdreich; Turnschuhsohle im Kalkmantel auf Sägemehl, dazu Teerholz - auf Plastiktüte und Stinkesocken; Heizölwein und scharfe Lauge + für die Kinder: Baumschwamm-Limonade“"55.

Auch beim regelmäßigen Familienritual des gemeinsamen Essens, das meist aus tranchierter Schuhsohle besteht, geht es keinesfalls gesittet und ruhig zu: Unter dem Tisch schnarcht der Großvater, während die Kinder sich gegenseitig Schlammknödel in den Mund werfen. Die Olchis widersetzen sich allen zivilisierenden Ge- und Verboten: Sie ,schmatzen und rülpsen, schlabbern und schlürfen, wie es sich für Olchis gehört" 56 . Um diese Geräuschkulisse und ihre Kleckereien zu Hause naturgetreu imitieren zu können, werden im Fanshop neben den abwaschbaren Tischdecken als Partyartikel unter anderem „Olchis Olchige Schlürfröhre“ und „Schlabberbecher“ angeboten ${ }^{57}$. Die fraglichen Manieren können so spielerisch im kontrollierten Raum eines „Kindergefurztags“ ausagiert werden. Ein Kinderfest wird zur emanzipatorischen Experimentalbühne, zu einem Frei- und Kompensationsraum ohne Regeln und Beschränkungen, in dem, wie Maria Lypp es formulierte, die triumphierende „Komik der Befreiung“ herrscht58. Auf den ersten Blick bestätigt sich hier der Trend der kinderliterarischen Werke, unfeine Essgewohnheiten und Tabuverletzungen zu fördern, den Annelore Enge bereits 1993 indigniert monierte ${ }^{59}$. Durch die

53. - Richter, Schlaraffenland, a.a.O., S.72.

54. - Dietl, Die Olchis, a.a.O., S. 79.

55. - Ebd., S. 117.

56. - Ebd., S. 28.

57. - Die Palette der Merchandising-Artikel ist enorm: Neben Büchern werden Spiele (Kartenspiele, Sudoku, Blockspiele), diverse Party- und Scherzartikel, Schulmaterial, Rucksäcke, Regenschirme, Malbücher, Zauberbedarf, Badespaß etc. angeboten. (www.oetinger.de/buecher/fanartikel-spielzeug/die-olchis/seite/2/5.html Abruf: 27.08.2015)

58. - Maria Lypp, Lachen, a.a.O., S. 91-93.

59. - Annelore Enge, „Das Land der Pfannekuchen und Pommes Frites? Über Essgewohnheiten und Tischgemeinschaften in Kinderbüchern“, in: Grundschule 5 
Ausmalung von Grobianismen wird neben der Imitation indessen auch eine moralisierend-karikierende Distanzierung angestrebt. Die verinnerlichten Konventionen ermöglichen es dem kindlichen Rezipienten, die Regelverstöße der Olchis als solche zu erkennen.

Mit ihrer sinnlichen Suggestivkraft zeigen die untersuchten Bilderbücher, dass die Machtkämpfe am Tisch als die „erste Kraftprobe zwischen den Erwachsenen und Kindern"60 im Rahmen eines Enkulturationsprozesses nichts an ihrer Brisanz eingebüßt haben. Nach wie vor bergen die zurückgewiesenen Speisen ein beträchtliches Konfliktpotential in sich; der Gestus der Verweigerung und die daraus resultierenden Repressionssituationen werden mit List oder kompensatorischen Phantasien gelöst. Dem Primat der Kindgemäßheit folgend, geht es dabei weniger um kulinarische Finessen und Exzesse, sondern um die Nutrition, den kreatürlichen Akt der Nahrungsaufnahme. Oft spiegeln die dargestellten Mahlzeiten eine tendenzielle Auflösung der traditionellen Familie als Tischgemeinschaft wider. Wiederum bestätigt das überlegene Lachen über die verlachenswerten Grobiane den gesellschaftlichen Konsens, der nach wie vor das normative comme-il-faut am Tisch festlegt.

(1993), S. 23-25.

60. - Könneker, Struwwelpeter, a.a.O., S. 165. 